\title{
Polymer Optical Fiber Modification by Etching using Hansen Solubility Parameters - A Case Study of TOPAS, Zeonex and PMMA
}

Inglev, Rune; Bang, Ole; Woyessa, Getinet; Janting, Jakob

Published in:

Journal of Lightwave Technology

Link to article, DOI:

10.1109/JLT.2019.2919798

Publication date:

2019

Document Version

Peer reviewed version

Link back to DTU Orbit

Citation (APA):

Inglev, R., Bang, O., Woyessa, G., \& Janting, J. (2019). Polymer Optical Fiber Modification by Etching using Hansen Solubility Parameters - A Case Study of TOPAS, Zeonex and PMMA. Journal of Lightwave Technology, 37(18), 4776 - 4783. https://doi.org/10.1109/JLT.2019.2919798

\section{General rights}

Copyright and moral rights for the publications made accessible in the public portal are retained by the authors and/or other copyright owners and it is a condition of accessing publications that users recognise and abide by the legal requirements associated with these rights.

- Users may download and print one copy of any publication from the public portal for the purpose of private study or research.

- You may not further distribute the material or use it for any profit-making activity or commercial gain

- You may freely distribute the URL identifying the publication in the public portal 


\title{
Polymer Optical Fiber Modification by Etching using Hansen Solubility Parameters - A Case Study of TOPAS, Zeonex and PMMA
}

\author{
Rune Inglev, Getinet Woyessa, Ole Bang and Jakob Janting
}

\begin{abstract}
Solvents can be used in the fabrication process of Polymer Optical Fiber (POF) sensors, as tapering or etching agents. We present a general approach - the use of Hansen Solubility Parameters (HSPs) - for identifying usable solvents for etching and present the first results on etching of TOPAS and Zeonex POFs. We also present alternatives to acetone in the form of trichloroethylene and THF, as etching solvents for PMMA, as well as results on the etching rate dependence on fiber orientation and annealing.
\end{abstract}

Index Terms-Polymer Optical Fibers, POF, Etching, TOPAS, Zeonex, PMMA, Hansen Solubility Parameters, HSP

\section{INTRODUCTION}

$\mathbf{P}$ OLYMER Optical Fibers (POFs) are alternatives to silicafibers within the realm of fiber optical sensors. They are interesting because they are amenable to modification by simple solvents or chemicals at different stages. They can, for example, be doped with fluorescent molecules at the fiber post-processing stage [1]-[3], or be surface-functionalized by simple chemistry [4]. POFs can also be etched or tapered locally in order to increase the performance of Fiber Bragg Grating (FBG) sensors [5]-[9], or solvents can be used as an alternative to annealing for stress relaxation of the polymer [10] - a crucial step in fabrication of stable sensors. This is contrary to silica-fibers, where etching is done using HF (Hydrofluoric Acid), a dangerous acid requiring cumbersome protective wear, and doping must be performed at the preform fabrication stage. There is also no good alternative to HF for silica-etching, but for thermoplastic polymers, due to the nature of the etching, there can be many possible solvents, or mixtures of solvents, which will do the job.

When speaking about polymers, "etching" really means controlled dissolution of the material, rather than the chemical reactions of the silica-HF system [11]. The process should preferably leave the etched region of the fiber with a smooth surface similar to the unetched region. Thus, when working with POFs, the need to understand which solvents are able to dissolve a given polymer is important, as not all solvents of a polymer will behave as "good" etchants. Hansen Solubility Parameters can help in this regard, as will be shown in this paper.

R. Inglev, O. Bang, G. Woyessa and J. Janting are with the Technical University of Denmark, Department of Photonics, 2800 Kongens Lyngby, Denmark (emails: ruing@fotonik.dtu.dk, oban@fotonik.dtu.dk, jajant@fotonik.dtu.dk, gewoy@fotonik.dtu.dk)

O. Bang is also with SHUTE Sensing Solutions A/S, Oldenvej 1A, 3490 Kvistgaard, Denmark
Solvent etching is also different from another technique, thermomechanical tapering, in several aspects. One obvious fact is that, for microstructured fibers, thermomechanical tapering will reduce the core-size, and thereby change the guiding properties of the fiber, whereas etching will merely remove the bulk material outside the rings, leaving the core untouched. Thermomechanical tapering will also change the properties of an FBG in the tapered region. Furthermore, it has been shown that solvent etching is superior in the fabrication of Compound Parabolic Concentrators (CPCs) [11]. However, etching the fiber is dependent on the history of the polymer material, and so, even though two fibers are of the same material, internal stresses and thermal history can account for differences in how they respond to etching, even with the same solvent. In contrast, thermomechanical tapering is more likely to be dependent on equipment setup and control, rather than internal fiber properties [12].

Currently, PMMA (poly(methyl methacrylate)) is a commonly used polymer for POFs, both as cladding and core material, and for both step-index and microstructured fibers. Acetone, either as a pure solvent or in a mixture, is a wellknown solvent for PMMA and can be used to remove the cladding of a PMMA-clad silica-fiber, or reduce the diameter locally of a POF [5], [7]-[9], [13]. As interest in other polymers gain traction, knowledge of similar solvents for these will be needed.

TOPAS and Zeonex are two such polymers, which are interesting for several reasons. Both Polymers have a slightly higher index of refraction than PMMA (TOPAS 5013S-04: 1.53, Zeonex 480R: 1.525, PMMA: 1.49 - all depending on grade), with optical losses comparable to PMMA. However, both Zeonex and TOPAS have a lower transmission edge in the UV part of the spectrum than PMMA, and could therefore be useful in cases requiring transmission of blue or violet light [14], [15]. Both the mentioned TOPAS and Zeonex grades can also withstand higher temperatures than typical commercial PMMA, due to higher glass transition temperatures [16] (TOPAS: $134{ }^{\circ} \mathrm{C}$, Zeonex: $139{ }^{\circ} \mathrm{C}$, PMMA: typically $105^{\circ} \mathrm{C}$, but unknown exactly for the type used in this paper) and FBG sensors made in TOPAS and Zeonex fibers are less sensitive to the humidity of the air [17]-[19].

What we want, is to be able to "etch" the polymer fiber using a given solvent, and as explained before, by "etching" we really mean "controlled dissolution", where the polymer is stripped away layer-by-layer. This could be as a first step in modification of the fiber, after which a layer of sensing 
material is applied to the etched fiber, or in order to increase the sensitivity of an FBG. See for example Janting et al. [9], in which a $\mathrm{pH}$ sensitive gel is applied to an etched section of the fiber, where an FBG has been inscribed, in order to increase the sensitivity of the sensor. Similarly, it is possible to fabricate Compound Parabolic Concentrators (CPCs) at the end of the fiber, to increase the collection of light. This has for example been done by Hassan et al. [11], [20], in which the CPCs are used to increase the collection of fluorescence from a biochemical sensing assay at the distal end of the fiber. Such a process requires that the solvent used produces an even and uniform material surface. Another common reason could be that one wishes to connect a POF with for example an FC or APC connector [21]. The drawn fiber may have a diameter somewhat larger than the connector hole, and using a suitable solvent, the diameter can be reduced until the fiber can be inserted.

Not all solvents for a given polymer will be able to create an even surface on the etched fiber, and what is of interest then, is to find "good" etching solvents. In this paper, we present solvents suitable for etching of TOPAS and Zeonex POFs, as well as revisit the etching of PMMA, by using Hansen Solubility Theory as a framework. Preliminaries of this work was first presented at the OFS-26 conference [22].

\section{THEORY}

\section{A. Hansen Solubility Theory}

The Hansen Solubility theory was developed by Charles Hansen in his Doctoral thesis from 1967 [23], [24]. In this framework, polymers and solvents are represented in a 3Dspace by three coordinates called the Hansen Solubility $\mathrm{Pa}$ rameters (HSPS). Each of the parameters are related to the strength of one of the long-distance cohesive forces - polar bonding energy density represented by $\delta P$, dispersive bonding energy density represented by $\delta D$ and hydrogen bonding energy density represented by $\delta H$. Distances in HSP space are calculated using the formula

$$
R_{a}=\sqrt{4 \Delta D^{2}+\Delta P^{2}+\Delta H^{2}}
$$

Where $\Delta D, \Delta P$ and $\Delta H$ are the differences in the $\delta D, \delta P$ and $\delta H$ parameters of the polymer and solvent.

For polymers (and pigments) an additional parameter termed the interaction/solubility distance, $R_{o}$, is also defined. Solvents within this distance are able to dissolve the polymer. Thus, for solvents with a distance $R_{a}<R_{0}$ to the polymer, dissolution of the polymer will occur. This means that polymers can be represented by ellipsoids in HSP Space, while solvents are represented by points. From a practical perspective, the interaction distance of a polymer, is a measure of how difficult it is to dissolve the polymer. A small distance, means that to dissolve the polymer the solvents used must have a very similar distribution of cohesive forces. Another way to term this is to say that "like dissolves like" - a well known aphorism. Another concept to mention is the RED value (Relative Energy Difference) given by $R E D=R_{a} / R_{o}$. For $R E D<1$ the solvent is within the ellipsoid, while an $R E D>1$ means the solvent is outside.
Over the years, the community using HSPs has compiled a large database for various solvents making Hansen Solubility theory an easy-to-use method in determining thermoplastic polymer solubility. It is also possible to mix the individual solvents HSPs - the mixture HSPs will then be an average of the solvents (by volume). The mixing of two or more nontoxic solvents is often used in finding alternatives to a toxic one.

The theory is mainly aimed at thermoplastic polymers, since thermosets will not dissolve in a solution, due to cross-links between the polymer chains.

\section{B. Determination of HSPs and $R_{o}$ for Polymers}

Determining the solubility parameters for a given polymer is done via a series of simple experiments. A piece of the polymer is dropped into a given solvent, and time is allowed to do its work. Solvents which dissolve the polymer are marked as "good" and solvents which did not work are marked as "bad". The time it takes for dissolution to occur, depends on a number of factors, such as stirring, size of the sample, the diffusivity of the solvents and $R E D$. Increasing the temperature can also decrease the time it takes for full dissolution, but the solvent HSPs are dependent on temperature, and so must be adjusted. [24].

After collecting the test results, a fitting algorithm is used to determine a set of HSPs and $R_{0}$ for the polymer. This can then be used when looking for alternatives to a solvent in the form of another compatible solvent or even blends of solvents - simply look up the HSPs of the solvent, or calculate it for the solvent mixture, and check if it is inside or outside the ellipsoid of interaction.

\section{MATERials \& Methods}

\section{A. Canes and Fibers}

Canes and fibers have been manufactured from raw materials at our department. The fibers are microstructured polymer optical fibers (POFs) made by the two-step drawing process first drawing the preform to canes, and then drawing the canes to fibers.

The TOPAS canes and fibers used in this paper have been manufactured locally at our department. The raw TOPAS pellets were acquired from the company TOPAS - Advanced Polymers [14], and the grade is 5013S-04 with a glasstransition temperature of $134^{\circ} \mathrm{C}$.

Zeonex canes and POFs were also manufactured at our department. Pellets were acquired from ZEON Corporation [15], and the grade is $480 \mathrm{R}$ with a $T_{g}$ of $139^{\circ} \mathrm{C}$.

PMMA was purchased as cylinders, in which the microstructure of the POFs was drilled at our department. The cylinders were then drawn to canes. The polymer is GEHR PMMA $^{\circledR}$

Before use in this study the Zeonex and PMMA fibers were annealed for at least 6 hours at $75{ }^{\circ} \mathrm{C}$. Annealing has been found in some cases to alleviate problems with crazing and cracking. The TOPAS fibers were etched both annealed and un-annealed. The annealing of TOPAS fibers were done by placing them in $110^{\circ} \mathrm{C}$ for 3 days. 
For each of the polymers, the canes were used in the determination of solubility parameters, and the fibers for the etching experiments.

\section{B. Chemicals}

Chemicals were of standard laboratory grade $(95 \%)$ or better and acquired through Sigma-Aldrich. The solubility parameters for the chemicals are from the software HSPiP version 5.0.09 [25]. Some of the chemicals used are toxic, and therefore caution should be used in evaluation of solubility parameters. As mentioned earlier, the theory allows one to find mixtures of solvents having the same HSP as another solvent. In this way, it would be possible to swap a toxic chemical for a mixture of two or more non-toxic chemicals. However, in our experiments, only single solvents are used, since the etching properties may be different for a mixture than for a single solvent, even if the mixture is designed to have the same solubility parameters as the single solvent. This will be further discussed in section VII.

When selecting chemicals for "etching", it is not enough to simply look for solvents with an $R E D<1$. We will look for solvents somewhat close to the edge of the ellipsoid, but still inside, while keeping an eye on the molar volume $(\mathrm{MVol})$ of the solvent. The $\mathrm{MVol}$ of the solvent will have some impact, especially in the kinetics of the process. We discuss this more in section VII.

It is worth noting something in regards to the purity of the solvents used. As mentioned, mixtures of solvents will produce HSPs for the mixture, which are an average (by volume) of the HSPs for the individual solvents. In a $95 \%$ pure solvent, the HSPs will be different than for a pure solvent. The actual HSPs will depend on what the remaining $5 \%$ consists of.

If we assume the $5 \%$ is water, the parameters for some solvents will be almost 1 unit different from the original value. The different HSPs of the impure solvent, will subsequently have an impact on the RED. A Monte-Carlo analysis has been performed, allowing the mixture to vary between 0 and $5 \%$ water content, and then calculating the corresponding RED values for the solvents. The analysis showed that some of the solvents, could have a difference in RED of about 0.15 units. This can obviously have an impact on the solubility if the solvent is close to the boundary. Thus, for the etching experiments, solvents of higher purity $(99 \%)$ or better were used, keeping the difference in $R E D$ to less than 0.04 for the worst cases. This still may have an impact in the cases where we are very close to the sphere, and must be kept in mind.

\section{Procedure for Determining HSP}

Annealed canes (ca. $5 \mathrm{~mm}$ diameter), were cut in pieces of approximately $0.2 \mathrm{~g}$. Each of the pieces are then dropped into an Erlenmeyer flask filled with one of the solvents to be tested. A magnet for continuous stirring throughout the test is also added and the flask sealed. The tests are performed at standard laboratory conditions, with ambient temperatures ranging from 20 to $25^{\circ} \mathrm{C}$. Each of the tests are then observed for up to 1 week, after which it will be known which solvents dissolve the polymer and which do not. This time is based upon experience. However, one could imagine some special solvents taking up to 2 weeks for dissolution.

Evaluation of the solvent is done on the basis of whether the final product is a homogeneous solution, as determined by visual inspection. The time it takes for the dissolution is not a parameter used in evaluation, since the kinetics of the dissolution process are governed by other factors, not related to the HSPs. See Hansen for a more in-depth discussion of this [24].

The results from the tests are then used together with the HSPiP software. The program will determine the best fit for the ellipsoid. There is some uncertainty associated with the fit - running the program again will tend to produce slightly different HSP and $R_{0}$ values. Adding more experimental data will likely also shift the HSP values slightly and change the interaction radius. The uncertainty is greatest near the boundary of the interaction ellipsoid - some solvents may be inside the ellipsoid, even though they do not dissolve the polymer, and vice versa. Having more tests will give better results in terms of fixing the ellipsoid to a center, but the behavior near the boundary, will still be uncertain, as other effects that start to have an impact (e.g. entropy and enthalpy [24]-[26]).

The fitting algorithm was run 20 times, an average was then calculated, as well as the standard deviation.

\section{Procedure for Etching Rate Determination}

The etchings are performed by filling beakers with the etching solvent and 5 fiber pieces are then immersed into the solvent, being kept in a vertical orientation, and fixed on a laboratory stand. No stirring of the solvent is used during the etching and no sealing used. At particular times a fiber piece is removed. A suitable solvent is used to rinse and clean the fiber (which must be miscible with the etching solvent), typically water or 2-propanol. The diameter is measured using a micrometer (Mitutoyo IP-65) with a resolution of $1 \mu \mathrm{m}$. For each of the five fibers, the etching rate (in terms of radius reduction) is calculated, and an average is taken. The tests are performed at standard laboratory conditions, with temperatures ranging from 20 to $25^{\circ} \mathrm{C}$.

\section{Solubility Results}

\section{A. Solubility Parameters for Polymers}

For each of the polymers studied, solubility experiments were performed. The HSPiP software was then used for fitting the data to an ellipsoid (fig. 1) and calculate the HSP and $R_{0}$ values. The results can be seen in Figs. 1a, 1b and 1c.

Fig. 1d shows all three polymers together. The sizes and positions in the HSP space is then easy to compare. From the figures and HSPs in table I, it is easy to see that TOPAS and Zeonex have their centers in the less polar volume of the space, and will thus tend to be more readily dissolved by nonpolar compounds. Their ellipsoids obviously extend up into the more polar part of the space, but their radii are small, and thus solvents with "polarities" of $\delta P>5.8$ (Zeonex) and $\delta P>3.97$ (TOPAS) will not be able to dissolve them. 


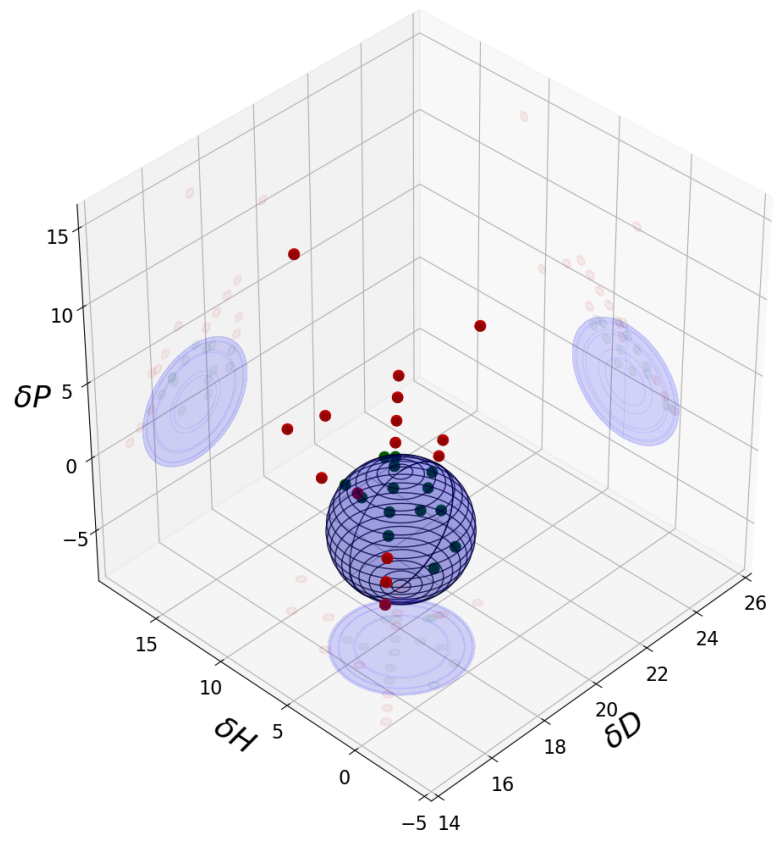

(a) Solubility ellipsoid of TOPAS.

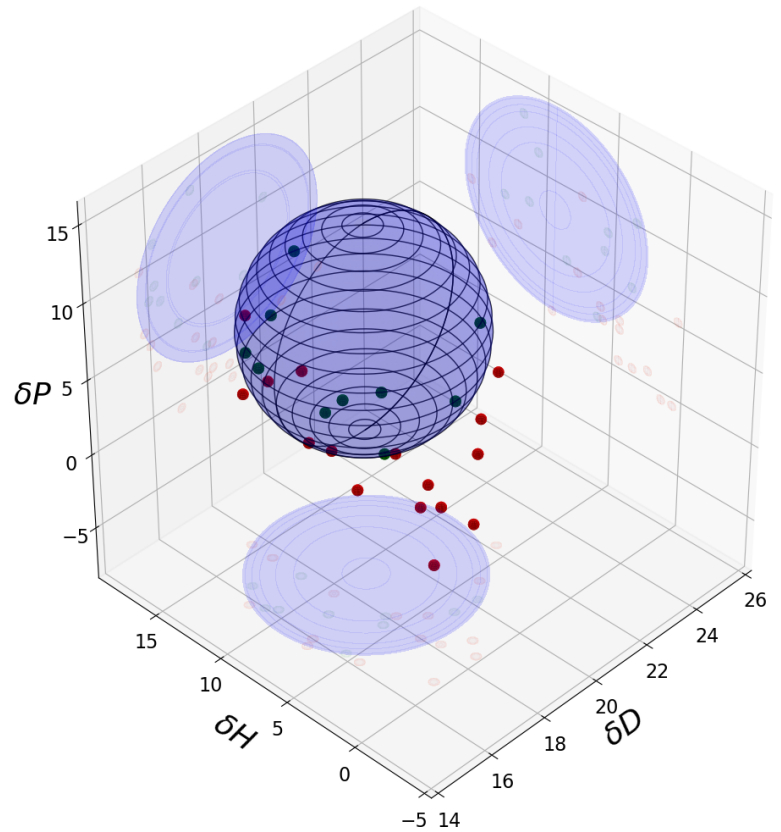

(c) Solubility ellipsoid of PMMA.

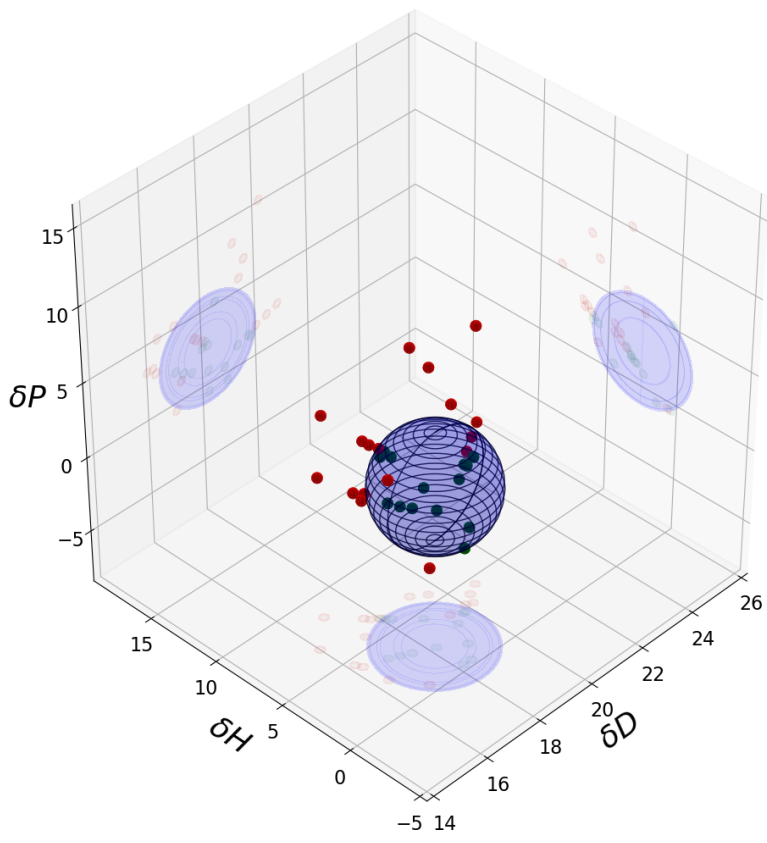

(b) Solubility ellipsoid of Zeonex.

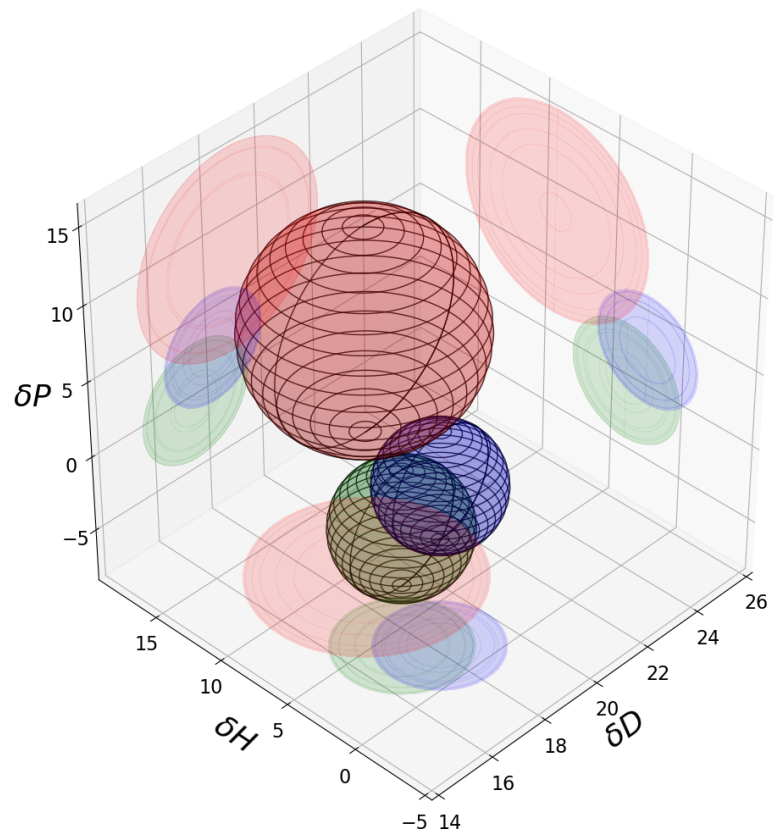

(d) Comparison figure.

Fig. 1: The sphere represents the interaction distance of the polymer, with the center of the sphere being the HSPs of the polymer. Each of the red and green points in figs. (a), (b) and (c) represents a solvent which has been tested - with red representing solvents that do not dissolve the polymer, while green represent ones that do. Negative values of the Hansen parameters are not physical, and that part of the space can be regarded as virtual. In the figs. (a), (b), (c) and (d), the axis for the dispersive bonding has been compressed, to provide a spherical view of the ellipsoid (a convention for HSP plots). Fig. (d) provides a comparison between the three polymers. Overlaps in the ellipsoids, will be regions where it is possible to find solvents (or mixtures) that dissolve all three polymers. The center position of the PMMA (red) compared to Zeonex (blue) and TOPAS (green) makes it clear that the two latter polymers are less polar, and will be dissolved by non-polar solvents more readily than PMMA. 
TABLE I: HSP Values for Polymers

\begin{tabular}{lcccc}
\hline Polymer & $\delta D(\sigma)$ & $\delta P(\sigma)$ & $\delta H(\sigma)$ & $R_{a}(\sigma)$ \\
\hline TOPAS & $17.23(0.09)$ & $0.02(0.01)$ & $3.37(0.21)$ & $3.95(0.05)$ \\
Zeonex & $18.23(0.19)$ & $2.76(0.12)$ & $2.09(0.07)$ & $3.66(0.14)$ \\
PMMA & $18.36(0.08)$ & $8.89(0.10)$ & $8.61(0.90)$ & $6.7(0.09)$ \\
\hline
\end{tabular}

Note 1: The units of the HSPs are $\left[\mathrm{MP}^{1 / 2}\right]$

Note 2: The standard deviation, $\sigma$, is in parentheses.

TABLE II: A list of selected solvents and corresponding RED

\begin{tabular}{lcccc}
\hline & \multicolumn{3}{|c|}{ RED } & \\
\hline Chemical & TOPAS & Zeonex & PMMA & MVol \\
\hline Acetone & $2.92(0.04)$ & $2.9(0.12)$ & $0.92(0.05)$ & 73.8 \\
1-Bromonaphthalene & $1.89(0.05)$ & $1.41(0.11)$ & $1.29(0.07)$ & 140.1 \\
n-Butyl Acetate & $1.4(0.04)$ & $1.78(0.1)$ & $1.15(0.05)$ & 132.6 \\
Chloroform & $1.02(0.04)$ & $1.03(0.05)$ & $0.99(0.06)$ & 80.5 \\
Cyclohexane & $0.83(0.05)$ & $1.21(0.08)$ & $1.89(0.09)$ & 108.9 \\
Di-Isobutyl Ketone & $1.14(0.03)$ & $1.36(0.1)$ & $1.25(0.08)$ & 177.4 \\
Dibromomethane & $2.06(0.04)$ & $1.73(0.07)$ & $0.5(0.07)$ & 69.8 \\
Dimethyl Formamide & $4.01(0.06)$ & $3.94(0.15)$ & $0.88(0.06)$ & 77.4 \\
Ethanol & $4.68(0.07)$ & $5.19(0.19)$ & $1.78(0.12)$ & 58.6 \\
Hexane & $1.46(0.05)$ & $2.05(0.12)$ & $2.12(0.09)$ & 131.4 \\
Tetrahydrofuran & $1.87(0.04)$ & $1.97(0.08)$ & $0.69(0.03)$ & 81.9 \\
Toluene & $0.63(0.04)$ & $0.41(0.05)$ & $1.5(0.09)$ & 106.6 \\
Trichloroethylene & $1.0(0.03)$ & $0.9(0.04)$ & $1.01(0.07)$ & 90.1 \\
p-Xylene & $0.39(0.04)$ & $0.61(0.05)$ & $1.45(0.08)$ & 121.1 \\
\hline
\end{tabular}

Note 1: The units of $M V o l$ are $\left[\mathrm{cm}^{3} / \mathrm{mewl}\right]$

Note 2: Several of the solvents are toxic, and caution should be exercised.

Note 3: The numbers in parentheses are the standard deviations.

Table I lists the polymer HSP results from the fitting, and shows an increasing distance of solubility, $R_{0}$, going from TOPAS to PMMA. The smaller solubility volume of Zeonex and TOPAS compared to PMMA will result in fewer solvents within the ellipsoid, and thus fewer candidates for etching.

\section{B. RED Values for Solvents}

From the spheroid it is then possible to calculate $R E D$ values for various solvents, also ones not used in the actual solubility testing of the polymer. In table II, a limited list of solvents and their corresponding $R E D$ values are presented, as well as their molar volumes. Using the means and standard deviations from Table I, the means and standard deviations for the solvents have also been calculated.

\section{Etching Results}

\section{A. Etching Solvent Candidates}

The relationship between the solvent parameters (RED, $M V o l$ and possibly others) and well controlled etching is at the moment unknown. But as explained earlier, we believe that choosing a solvent close to the ellipsoid surface (but inside) and not a too small or too large molecular size are the important parameters in a first-order approximative sense. So we are limited to a trial-and-error approach.

For PMMA, the predicted solvents were acetone, chloroform, dibromomethane (DBM), dimethyl formamide (DMF), tetrahydrofuran (THF) and trichloroethylene. Trichloroethylene, even though shown to have an $R E D$ of 1.01 , we know to be a solvent for PMMA, and it also is within the uncertainty of 0.07 .

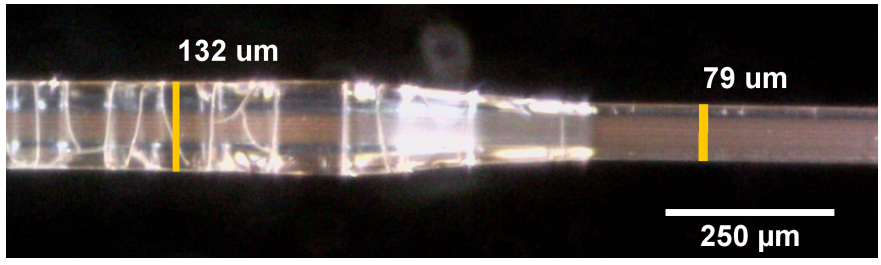

Fig. 2: Acetone etching of PMMA POF (annealed). The fiber was immersed in acetone for 6 minutes and the initial diameter was $132 \mu \mathrm{m}$ while the etched fiber was $79 \mu \mathrm{m}$. Cracks are visible in the part which were just above the solvent.

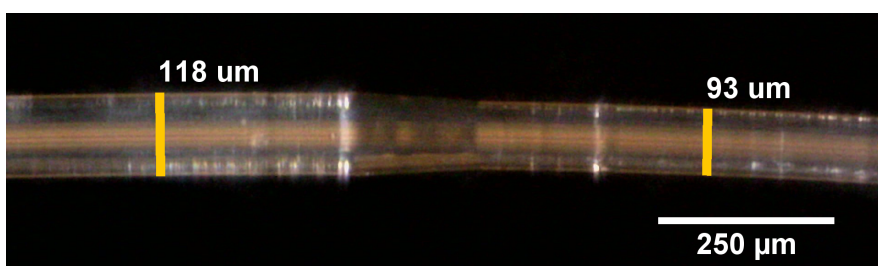

Fig. 3: Etching of PMMA POF (annealed) using trichloroethylene. The fiber was immersed for 2.5 minutes. The initial diameter was $118 \mu \mathrm{m}$ and the final diameter is $93 \mu \mathrm{m}$.

Likewise for TOPAS, cyclohexane, chloroform, toluene, trichloroethylene and p-xylene were tested. Chloroform and trichloroethylene are taken as candidates, with the same argument as for PMMA, that the uncertainty brings them within the ellipsoid.

Similarly, candidates for Zeonex were chloroform (again the uncertainty brings it inside), toluene, trichloroethylene and pxylene.

\section{B. Etching of PMMA POFs}

From our experiments it was found that acetone, trichloroethylene and THF are possible etchants for PMMA. We mentioned in the introduction that acetone has been used before to taper POFs locally, either in mixtures or as a pure solvent. Some researchers find pure acetone to induce crazing, see e.g. Merchant et al. [13], but at our department, this has not always been a problem. Possible reasons for this will be discussed in section VII. Acetone showed an etching rate of $4.3 \mu \mathrm{m} / \mathrm{min}$ and an example can be seen in Fig. 2. However, we did see some cracking at the portion of the fiber that had been just above the solvent surface. The acetone vapors seem to induce this cracking. We believe the explanation is a cooling of the part of the fiber just above the solvent, due to the cooling effect of evaporation (more details are given in section VII).

With trichloroethylene the annealed PMMA fibers were etched with a rate of $5.4 \mu \mathrm{m} / \mathrm{min}$. An example is shown in Fig. 3.

THF etched with a rate of $0.4 \mu \mathrm{m} / \mathrm{min}$ and was thus the slowest. This may be useful for situation in which precise control of the etching is required.

From the experiments it was found that chloroform is aggressive to PMMA and causes crazing, resulting in a milkywhite surface. DMF also induced crazing, and DBM acted very quickly, etching approximately $25 \mu \mathrm{m} / \mathrm{min}$ (calculated from a single fiber immersed for 1 minute). A more precise etching rate was not determined for DBM. 


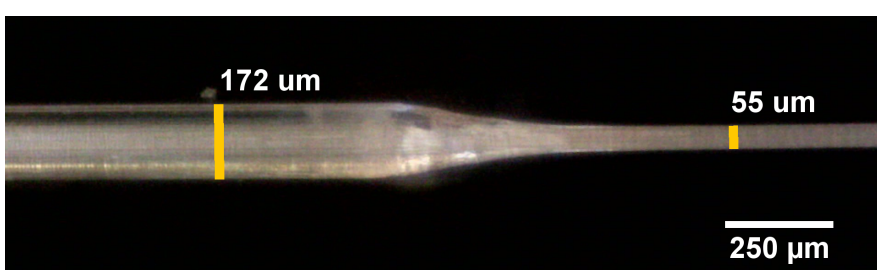

Fig. 4: A TOPAS POF (un-annealed) has here been immersed in cyclohexane for 5 minutes. The fiber diameter is reduced from $172 \mu \mathrm{m}$ to $55 \mu \mathrm{m}$.

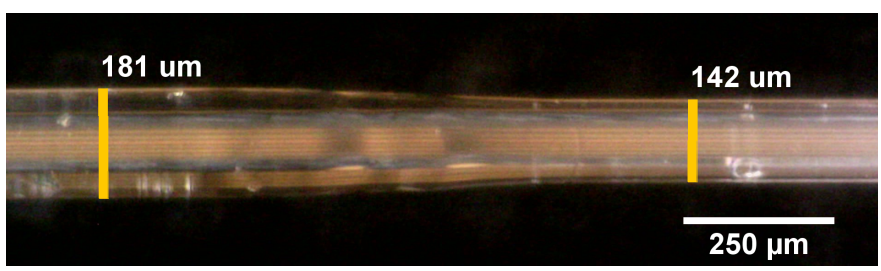

Fig. 5: Etched Zeonex POF (annealed). The fiber has been immersed in trichloroethylene for 6 minutes. The fiber diameter was etched from $181 \mu \mathrm{m}$ to $142 \mu \mathrm{m}$.

\section{Etching of TOPAS POFs}

For TOPAS, cyclohexane was the obvious candidate with an $R E D$ of 0.83 , and we found that it is indeed a good etchant. It etched the unannealed fibers with a rate of $8.9 \mu \mathrm{m} / \mathrm{min}$, and an example from that experiment is shown in Fig. 4. Earlier studies, with annealed fibers, also in a vertical orientation, gave an etching rate of $6.8 \mu \mathrm{m} / \mathrm{min}$.

Toluene, p-xylene, chloroform and trichloroethylene were also tested, however, they were very aggressive to the TOPAS polymer, and made it very rubbery. This can possibly be explained by a large uptake of the solvent, with a subsequent plasticizing effect [8].

\section{Etching of Zeonex POF}

There were several solvents tested with Zeonex. Toluene and p-xylene made the polymer very rubbery, and were therefore not good etchants. Chloroform dissolved the polymer too quickly, and was disregarded for further study.

However trichloroethylene showed good promise, and testing it revealed an etching rate for the annealed fibers of $3.3 \mu \mathrm{m} / \mathrm{min}$. In Fig. 5, one of the fibers from that experiment is shown.

\section{Vi. Etching Rate Dependence on ORientation And ANNEALING}

A small investigation on the etching rate dependence on various parameters has also been performed. $0.5 \mathrm{~mm}$ solid-core TOPAS fibers without cladding were etched in one of three different configurations, 1) Vertical orientation (in a beaker containing the solvent), 2) Horizontal orientation (in a petridish with local part of fiber isolated by UV-cured adhesive (DY MAX 9-318-F)), and 3) Horizontal orientation (with annealing). These were all performed with cyclohexane.

From the data also depicted in fig. 6, it is clear that there are significant differences in the etching rate, depending on orientation and annealing. Vertical orientation with an unannealed fiber results in the largest etching rate. Having the

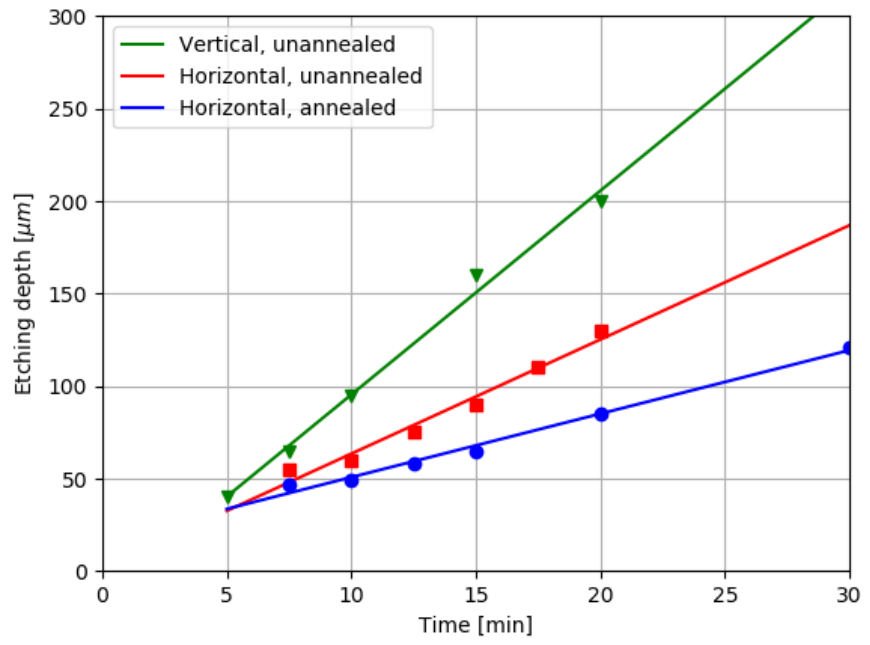

Fig. 6: Etching depth vs. time on a TOPAS fiber. The etching is performed in cyclohexane. Measurement was performed with a micrometer (Mitutoyo IP-65), with a resolution of $1 \mu \mathrm{m}$. For the green curve, the rate is approx. $10 \mu \mathrm{m} / \mathrm{min}$, which is comparable to the rate we found for TOPAS in section $\mathrm{V}-\mathrm{C}$.

fiber in a horizontal orientation reduces the rate significantly. The reason for this may be due to a convective effect, where the solvent-polymer liquid just at the interface has a higher density than the surrounding solvent, and gravity acts to pull it down along the fiber. This has also been investigated by Unger et al. [27]. Cyclohexane has a density of $0.78 \mathrm{~g} / \mathrm{ml}$, while TOPAS 5013 has a density of $1.02 \mathrm{~g} / \mathrm{ml}$, and so this convective effect could take place in this case.

If we also anneal the fibers (for this experiment it was performed for 66 hours at $110{ }^{\circ} \mathrm{C}$ ) the rate is also reduced. This is likely to do with the stress in the fiber. Since the solubility is a function of the relative energy difference between the solvent and the polymer cohesive forces, stress will be an added energy available to the process of dissolution. This extra energy then takes its form as a quickening of the process kinetics. Annealing is an important process to be mindful of when working with polymer optical fibers, as it can cause drift in FBG resonances, but also improve the performance [8], [17], [28].

\section{DISCUSSION}

The dissolution process is a complex phenomena involving two transport processes - solvent diffusion into the polymer, and polymer chain disentanglement [29]. The general picture of dissolution is that the polymer, during the process, can be roughly described by six layers as depicted in fig. 7. The liquid layer contains a high concentration of free polymer chains, that are in the process of diffusing into the solvent environment, while the gel-layer is a rubbery and swollen layer, still containing entangled chains. The solid swollen layer is a part of the polymer which has had solvent molecules diffuse into the matrix, and thereby inducing swelling, but the polymer is still in a solid state. The infiltration layer is the part of the polymer, in which the solvent molecules have moved into the free volume, consisting of molecular size holes and 


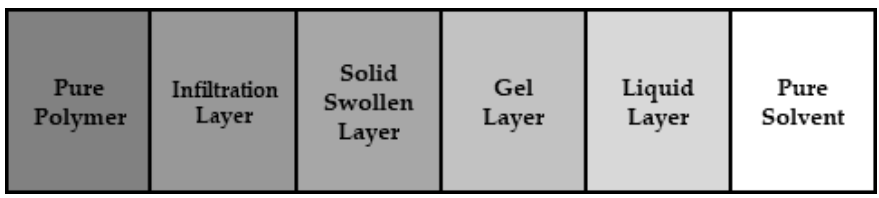

Fig. 7: The six layers defined during dissolution (reworked from Miller-Chou and Koenig [29]).

pores. The size of the different layers depend upon both the polymer, the solvent and temperature [29].

One of the primary aims of this study was to find solvents producing a homogeneous etch, where the etched part of the polymer, retains a smooth surface. If too large a gel-layer is formed, and the fiber is removed from the solvent, this rubbery part may then dry and form an uneven surface of the fiber. It is therefore of interest to find solvents that do not induce a thick gel-layer. The gel-layer can to some extent be removed by stirring [13], [29], however the etching setup may not allow for this in some cases, and so, finding solvents with minimal gel-layer formation is of interest.

On the opposite side of the spectrum, it has been found that if no gel-layer is formed, cracks may appear in the polymer, leading to dissolution by "eruption" of small pieces of polymer. [29]. In such cases, when removing the fiber from the solvent, the fiber surface would be cracked and crazed, leading to significant transmission losses, or the fiber may simply break apart due to the damage. We believe this may explain the cracking we saw for the PMMA fiber (see Fig. 2). So it is clear, that some intermediate region should be found, where a small or insignificant gel-layer is formed.

The diffusivity of the etching molecules into the polymer also has an impact on the etching. If the molecules are small and good solvents, they may tend to cause crack initiation, as investigated by Ouano and Carothers (referenced in MillerChou and Koenig [29]). However, the size of the solvent molecule will also impact the kinetics of the process, with increasing dissolution rate for smaller molecules. This has been the reason that we have initially been keeping an eye on the $\mathrm{MVol}$ of the solvent. Diffusion of the solvent into the polymer could also have an effect on the transmission through the fibers. Absorbed solvent can stay in the polymer for a long time, as was shown by Janting et al. [8].

Another contributor to the dissolution dynamics is the molecular weight and its distribution. It has been shown that the dissolution rate of a polymer decreases with higher molecular weights of the polymer. Additionally, the dispersity of the polymer will also have an impact, with some results showing that polydisperse polymers dissolved twice as fast as the monodisperse (referenced in Miller-Chou and Koenig [29]).

We also mentioned that acetone is often found by others to induce crazing of the surface, while we at our department have not encountered this problem. The problem we saw with the cracking we believe is caused by the acetone vapors. The etched portion of the fiber showed no damage. Possible explanations for this could be in terms of the molecular weight or the temperature of the polymer. As described in Miller-
Chou and Koenig [29], the thickness of the gel-layer was found to decrease, as the temperature was lowered from the glasstransition temperature to some critical temperature (termed the gel-temperature). Below this temperature, polymers start to exhibit dissolution by cracking and "eruption". It is possible, that the gel-temperature will be different, if the $T_{g}$ of the polymer is changed. Thus, for two samples of PMMA, one having a $T_{g}$ lower than the other, their gel-temperatures may also be different. Since $T_{g}$ of a polymer is related to the molecular weight, through the Flory-Fox Equation [30], a lower molecular weight would result in a lower $T_{g}$ and a possibly lower gel-temperature. The gel-temperature may then be below the processing temperature, in which case cracking would cease.

Another explanation may be related to the stresses in the polymer. If the polymer is not stress-relaxed, residual surface stresses from the fiber-drawing could be a player in inducing crazing and cracking of the surface. This was also mentioned in section III-A.

A last thing to mention is about the use of mixtures. In section III-B, we mentioned that we would not be using mixtures of solvents. The overall behavior of the dissolution using a mixture, is governed by the mixture HSPs, and as with single solvents, if the $R E D<1$, the mixture will be able to dissolve the polymer. However, the dynamics of the dissolution process with a mixture is different than for individual solvents. Each of the components in the mixture, will have a specific diffusivity in the polymer, and so, one of the components may diffuse into the polymer more readily than the other. This may lead to effects not seen with an individual solvent with the same HSPs as the mixture. For example, it was shown by Cooper et al. (referenced in Miller-Chou and Koenig [29]) that the addition of small non-solvent molecules to a good solvent, could increase the dissolution rate. The reason was thought to be because of plasticization of the polymer by the small molecules. What this means for our study is that there may be effects from the interplay between the two solvents, that affect the etching, which cannot be seen as a result of either the RED of the mixture or its HSPs. Further studies are required to determine how mixtures behave as etching solvents for polymers.

\section{CONCLUSION}

We presented, in this paper, Hansen Solubility theory as a tool to help in the selection of etching solvents for POFs. Cyclohexane was found to be a good solvent for etching of TOPAS POFs, while trichloroethylene was found to be a possible agent for Zeonex. Acetone (pure and in mixtures) has long been used in etching of PMMA, and we showed that trichloroethylene and THF were likewise good etching solvents.

The exact selection criteria, when looking at solvent parameters such as $\mathrm{MVol}$ and HSPs, for when a solvent will behave as a "good" etchant, is still unknown, and many questions remain. However we found that an RED within the range of $0.7-1$ is a good guideline. We also discussed how "good" etching can be seen in terms of general dissolution behavior, such as the 
gel-layer formation. We also showed how the orientation and annealing of a fiber during etching has significant impact on the etching rate.

The approach using HSP can be used for any polymer of interest to the POF researcher, and is a valuable tool when one wishes to find alternatives to specific chemicals for polymer solubility.

\section{ACKNOWLEDGMENTS}

This work has been funded by The European Maritime and Fisheries Fund and The Danish Fisheries Agency.

\section{REFERENCES}

[1] M. C. J. Large, S Ponrathnam, A Argyros, N. S. Pujari, and F Cox. Solution doping of microstructured polymer optical fibres. Optics Express, 12(9): 1966, 2004.

[2] C. Pulido and Ó Esteban. Tapered polymer optical fiber oxygen sensor based on fluorescence-quenching of an embedded fluorophore. Sensors and Actuators B: Chemical, 184:64-69, 2013.

[3] Pavol Stajanca, Ievgeniia Topolniak, Samuel Pötschke, and Katerina Krebber. Solution-mediated cladding doping of commercial polymer optical fibers. Optical Fiber Technology, 41:227-234, 2018.

[4] Grigoriy Emiliyanov, Jesper B. Jensen, Ole Bang, Poul E. Hoiby, Lars H. Pedersen, Erik Michael Kjær, and Lars Lindvold. Localized biosensing with Topas microstructured polymer optical fiber. Optics Letters, 32(5):460, mar 2007.

[5] Ginu Rajan, Bing Liu, Yanhua Luo, Eliathamby Ambikairajah, and Gang-Ding Peng. High Sensitivity Force and Pressure Measurements Using Etched Singlemode Polymer Fiber Bragg Gratings. IEEE Sensors Journal, 13(5):1794-1800, 2013.

[6] Wei Zhang, Xianfeng Chen, Chen Liu, Yuanfu Lu, Marcos Cardoso, and David J. Webb. Improved response time of laser etched polymer optical fiber Bragg grating humidity sensor. In Hypolito J. Kalinowski, José Luís Fabris, and Wojtek J. Bock, editors, 24th International, 2015.

[7] Ginu Rajan, Kishore Bhowmik, Jiangtao Xi, and Gang-Ding Peng. Etched Polymer Fibre Bragg Gratings and Their Biomedical Sensing Applications. Sensors, 17(10):2336, 2017.

[8] Jakob Janting, Jens Pedersen, Rune Inglev, Getinet Woyessa, Kristian Nielsen, and Ole Bang. Effects of Solvent Etching on PMMA Microstructured Optical Fiber Bragg Grating. Journal of Lightwave Technology, PP(c):1-1, 2019.

[9] Jakob Janting, Jens Pedersen, Getinet Woyessa, Kristian Nielsen, and Ole Bang. Small and Robust All-Polymer Fiber Bragg Grating based $\mathrm{pH}$ Sensor. Journal of Lightwave Technology, PP(1):1-1, 2019.

[10] Andrea Fasano, Getinet Woyessa, Jakob Janting, Henrik K Rasmussen, and Ole Bang. Solution-Mediated Annealing of Polymer Optical Fiber Bragg Gratings at Room Temperature. IEEE Photonics Technology Letters, 29(8):687-690, 2017.

[11] Hafeez Hassan, Ole Bang, and Jakob Janting. Polymer Optical Fiber Tip Mass Production Etch Mechanism to Achieve CPC Shape for Improved Biosensor Performance. Sensors, 19(2):285, jan 2019.

[12] Hafeez U1 Hassan, Jakob Janting, Soren Aasmul, and Ole Bang. Polymer Optical Fiber Compound Parabolic Concentrator fiber tip based glucose sensor: in-Vitro Testing. IEEE Sensors Journal, 16(23):1-1, 2016.

[13] D.F Merchant, P.J Scully, and N.F Schmitt. Chemical tapering of polymer optical fibre. Sensors and Actuators A: Physical, 76(1-3):365371, 1999.

[14] TOPAS Advanced Polymers. www.topas.com, 2019.

[15] ZEON Corporation. www.zeonex.com, 2019.

[16] Christos Markos, Alessio Stefani, Kristian Nielsen, Henrik K Rasmussen, Wu Yuan, and Ole Bang. High-Tg TOPAS microstructured polymer optical fiber for fiber Bragg grating strain sensing at 110 degrees. Optics express, 21(4):4758-65, 2013.

[17] Wu Yuan, Lutful Khan, David J. Webb, Kyriacos Kalli, Henrik K. Rasmussen, Alessio Stefani, and Ole Bang. Humidity insensitive TOPAS polymer fiber Bragg grating sensor. Optics Express, 19(20):19731, 2011.

[18] Getinet Woyessa, Andrea Fasano, Alessio Stefani, Christos Markos, Kristian Nielsen, Henrik K. Rasmussen, and Ole Bang. Single mode step-index polymer optical fiber for humidity insensitive high temperature fiber Bragg grating sensors. Optics Express, 24(2):1253, 2016.
[19] Getinet Woyessa, Andrea Fasano, Christos Markos, Alessio Stefani, Henrik K. Rasmussen, and Ole Bang. Zeonex microstructured polymer optical fiber: fabrication friendly fibers for high temperature and humidity insensitive Bragg grating sensing. Optical Materials Express, 7(1):286, jan 2017

[20] Hafeez Ul Hassan, Kristian Nielsen, Soren Aasmul, and Ole Bang. Polymer optical fiber compound parabolic concentrator tip for enhanced coupling efficiency for fluorescence based glucose sensors. Biomedical Optics Express, 6(12):5008, 2015.

[21] Ada Abang and David J. Webb. Demountable connection for polymer optical fiber grating sensors. Optical Engineering, 51(8):080503-1, 2012.

[22] Rune Inglev, Jakob Janting, Kristian Nielsen, Getinet Woyessa, and Ole Bang. The Application of Hansen Solubility Parameters for Local Etching of TOPAS Polymer Optical Fibers. In 26th International Conference on Optical Fiber Sensors, page TuE66. OSA, 2018.

[23] Charles M Hansen. The Three Dimensional Solubility Parameter and Solvent Diffusion Coefficient. Their Importance in Surface Coating Formulation, 1967

[24] Charles M. Hansen. Hansen Solubility Parameters: A User's Handbook. CRC Press, 2nd edition, 2007.

[25] Hansen Solubility Website. www.hansen-solubility.com, 2019.

[26] Manuel J. Louwerse, Ana Maldonado, Simon Rousseau, Chloe MoreauMasselon, Bernard Roux, and Gadi Rothenberg. Revisiting Hansen Solubility Parameters by Including Thermodynamics. ChemPhysChem, 18(21):2999-3006, 2017.

[27] M. A. Unger, D. A. Kossakovski, R. Kongovi, J. L. Beauchamp, J. D. Baldeschwieler, and D. V. Palanker. Etched chalcogenide fibers for nearfield infrared scanning microscopy. Review of Scientific Instruments, 69(8):2988-2993, 1998

[28] Getinet Woyessa, Kristian Nielsen, Alessio Stefani, Christos Markos, and Ole Bang. Temperature insensitive hysteresis free highly sensitive polymer optical fiber Bragg grating humidity sensor. Optics Express, 24(2): 1206, 2016

[29] Beth A. Miller-Chou and Jack L. Koenig. A review of polymer dissolution. Progress in Polymer Science, 28(8):1223-1270, 2003.

[30] Thomas G Fox and Paul J Flory. Second-Order Transition Temperatures and Related Properties of Polystyrene . I . Influence of Molecular Weight. Journal of Applied Physics, 21(581), 1950. 